\title{
MICROSURGICAL TESTICULAR DENERVATION FOR THE TREATMENT OF CHRONIC TESTICULAR PAIN - INITIAL RESULTS
}

\author{
Rafael Garcia de Oliveira, ${ }^{\mathrm{I}}$ Cesar Camara, ${ }^{\mathrm{II}}$ João de Magalhães Avancini Ferreira

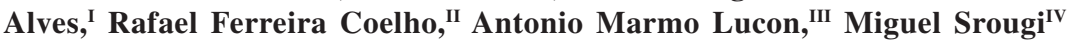 \\ doi: 10.1590/S1807-59322009000500004
}

de Oliveira RG, Câmara C, Alves J de MAF, Coelho RF, Lucon AM, Srougi M. Microsurgical testicular denervation for the treatment of chronic testicular pain - initial results. Clinics. 2009;64(5):393-6.

INTRODUCTION: Chronic testicular pain remains an important challenge for urologists. Currently, the treatment plan is primarily empirical, with the first approach consisting of clinical measures. However, some patients remain in pain despite a conservative treatment protocol and, for them, it is possible to perform a surgical procedure that involves severing the scrotal and spermatic branches of the genitofemoral and ilioinguinal nerve fibers.

METHODS: In our institution, 60 patients were evaluated and treated for idiopathic chronic testicular pain between January 2003 and July 2007. Priority was give to clinical treatment, which evolved from simple to more complex measures. Microsurgical treatment was performed on those who experienced no considerable pain relief (10 individuals in our study).

RESULTS: Over a twenty-four-month follow-up period, $70 \%$ of patients showed complete remission and $20 \%$ exhibited partial relief from pain.

KEYWORDS: Testicular diseases; Pain; Denervation; Microsurgery; Urologic surgical procedures.

\section{INTRODUCTION}

Chronic testicular pain (CTP) is defined as a continuous or intermittent, unilateral or bilateral testicular discomfort for at least three months that forces the patient to seek medical assistance. ${ }^{1}$ This condition is frequently presented as an important dilemma to both the patient and the doctor, who has great difficulty in determining a course of treatment.

In order to adequately deal with CTP, priority is given first to clinical treatment since surgical treatments such as orchiectomy and epididymectomy are associated with

\footnotetext{
'Hospital das Clínicas da Faculdade de Medicina da Universidade de São Paulo, São Paulo/SP, Brazil.

"Uro-Oncology Sector, Hospital das Clínicas da Faculdade de Medicina da Universidade de São Paulo, São Paulo/SP, Brazil.

IIIGeneral Urology Sector, Faculdade de Medicina da Universidade de São Paulo, São Paulo/SP, Brazil.

IV Department of Urology, Hospital das Clínicas da Faculdade de Medicina da Universidade de São Paulo, São Paulo/SP, Brazil.

Email: c-camara@uol.com.br

Tel.: 551130698080

Received for publication on November 20, 2008

Accepted for publication on February 03, 2009
}

variable success rates and are considered controversial. ${ }^{1,2,3}$ A careful study of the anatomy shows that innervation of the testicular organs is mediated by the scrotal and spermatic branches of the genitofemoral and ilioinguinal nerves, as well as by the sympathetic fibers that accompany the testicular artery. Based on this anatomy, some authors have proposed, with exciting preliminary results, a complete microsurgical transection of these nerve fibers for the treatment of CTP., ${ }^{4,5,6,7}$

The aim of this paper is to describe our treatment of 60 patients with chronic orchialgia and to present our results from microsurgical testicular denervation in 10 patients at our institution.

\section{PATIENTS AND METHODS}

Between January 2003 and July 2007, 60 patients were treated at our institution for idiopathic CTP. The mean age was 42 years (ranging from 22 to 68). All patients reported either continuous or intermittent testicular pain that had been affecting their quality of life for at least three months. 
All of the patients underwent a Doppler ultrasonography of their testes and urinary tract, urinalysis, urine culture and spermogram in order to rule out primary or secondary causes of pain, including intratesticular abscesses, tumors or uretheral lithiasis. We were unable to establish an etiological diagnosis for the testicular pain in any of the patients.

Our chosen clinical approach involved sequentially assigning simple and then increasingly more complex types of treatment without discontinuing previous regimens. These included the administration of commonly used analgesics, anti-inflammatory drugs and opioids, scrotal suspensory and/or corticoid injections in the spermatic cord, and the administration of tricyclic antidepressants. Psychological support was mandated after administering the antidepressants, and acupuncture was the last therapeutic resource employed. The pain intensity was controlled through the "Wong-Baker FACES Pain Rating Scale" (Figure 1), which ranked the pain from 0 to 5 . The patient's self-report was recorded and classified as worse, the same or better in comparison with the pain referred before the last consultation.

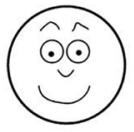

No Hurt

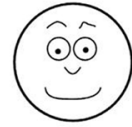

1 Hurst

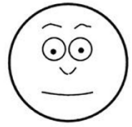

2 Hurts
Little More

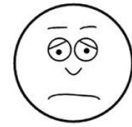

3 Hurts

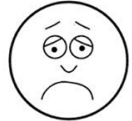

4 Hurts
Whole Lot

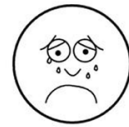

5 Hurst
Figure 1 - Wong-Baker FACES Pain Rating Scale

Those patients who did not respond to clinical treatment were submitted to a blockade of the spermatic cord with $0.5 \%$ lidocaine, and microsurgical testicular denervation was performed for those who presented with a resolution of the pain after the blockade.

Surgical procedure- Microsurgical Testicular Denervation: All the procedures were performed with the patient under spinal anesthesia. A $3 \mathrm{~cm}$ inguinal incision was made just above the inguinal ligament (Figure 2). The aponeurosis of the external oblique muscle was opened, and the ilioinguinal nerve was sectioned. The spermatic cord was repaired (Figure 3) and, using microscope magnification, 3 lymphatic vessels as well as the ductus deferens with its artery and vein were separated and preserved (Figure 4). The testicular artery was then identified and dissected by approximately $1.5 \mathrm{~cm}$, thereby blocking the sympathetic innervation juxtaposed to that vessel. After these steps, all the fibers of the cremasteric muscle - where the genital branch of the genitofemoral nerve runs - were sectioned. Intra-operative Doppler ultrasonography was not used to identify the testicular artery in our patients.

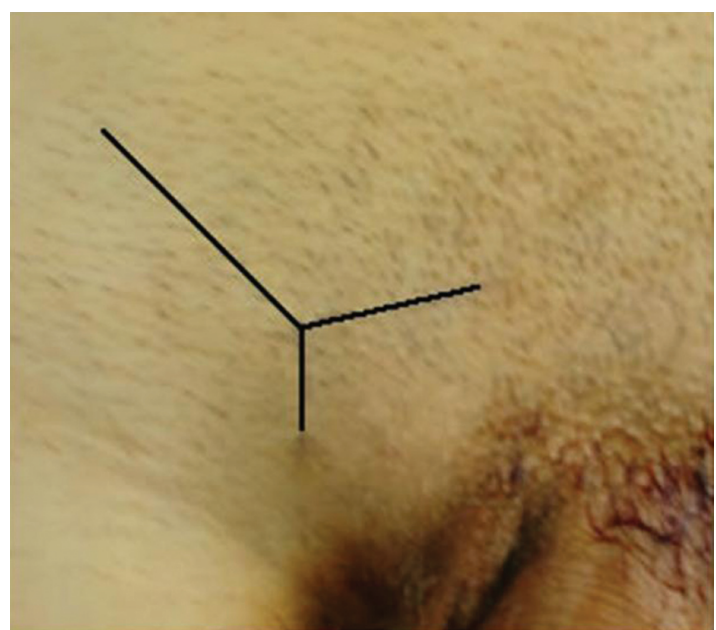

Figure 2 - Incision location

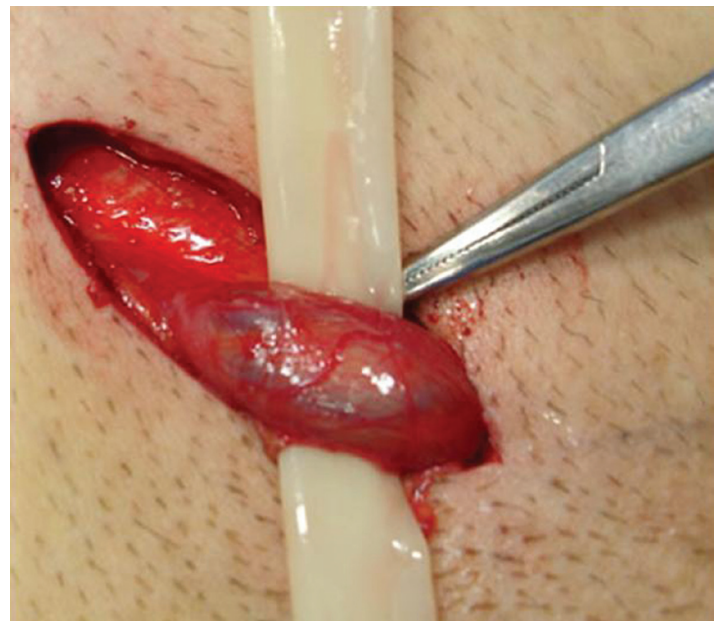

Figure 3 - Spermatic cord dissected and repaired

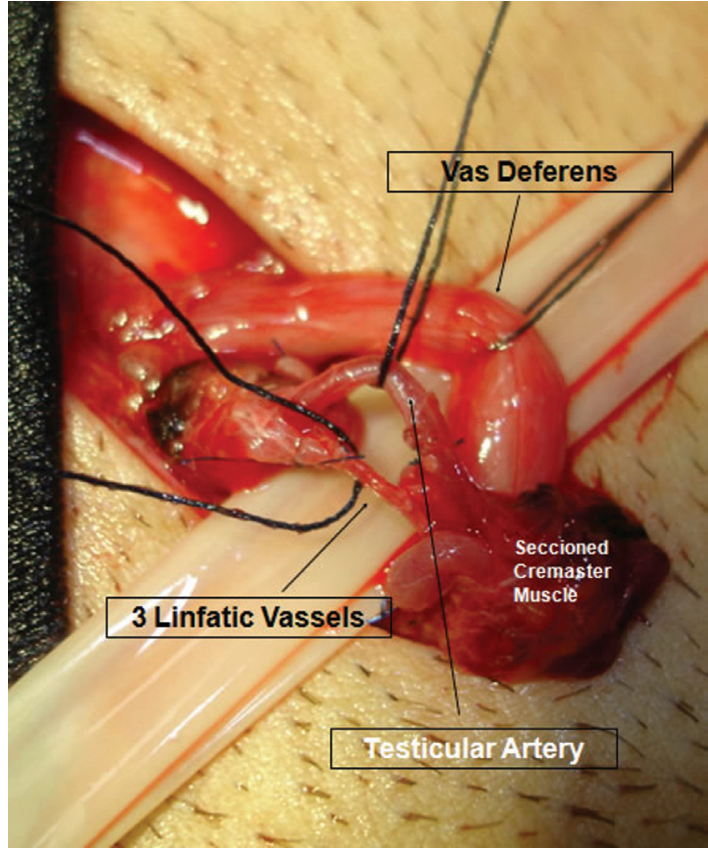

Figure 4 - View after microdissection 


\section{RESULTS}

All of the 60 patients included in our study had already consulted at least 3 doctors before their first evaluation at our institution. The mean duration of the pain was 48 (ranging from 3 to 73) months. Treatment with additional therapies showed only $10 \%$ adherence rates after 6 months of followup. Twenty-five patients were referred to acupuncture and treated with satisfactory results for 2 months. Despite the multiple clinical therapeutic modalities used, 10 out of 60 patients did not experience a satisfactory reduction in their pain. Thus, these patients requested more effective treatments to improve their quality of life. These patients were submitted to a blockade of the spermatic cord, which resulted in a resolution of the pain, and subsequently underwent microsurgical testicular denervation.

The mean surgical time was 90 minutes and we did not observe any intra or postoperative complications. Pain relief was assessed 4, 6 and 24 months after surgery. Four months after the procedure, six patients $(60 \%)$ reported complete remission of the pain. Six months later, seven patients $(70 \%)$ reported cure and two (20\%) reported improvement in their pain levels. Over a twenty-four-month follow-up period, the results did not change $-70 \%$ of patients showed complete remission and 20\% showed partial pain relief (Table 1).

Table 1. Surgically treated patients

\begin{tabular}{lll}
\hline Number of patients & & 10 \\
Age & & $33(26$ to 52$)$ years \\
Testicular units & & 10 \\
Follow-up & & $24(12$ to 42$)$ months \\
Success & Complete & $7(70 \%)$ \\
& Partial & $2(20 \%)$ \\
& No relief & $1(10 \%)$ \\
\hline
\end{tabular}

\section{DISCUSSION}

This study has shown that microsurgical testicular denervation may be used as a definitive treatment for patients with refractory CTP with a success rate of $90 \%$.

Despite the fact that CTP is a frequent complaint, there have been no studies to date regarding its prevalence among the male population. CTP can be idiopathic or may result from prior conditions such as testicular infection, trauma, varicocele or previous surgical interventions. In vasectomies, Ahmed et al. have found that up to $0.5 \%$ of all patients may develop CTP ${ }^{1}$. However, even after extensive investigation, up to $25 \%$ of all CTP patients cannot be assigned an etiological diagnosis for their orchialgia. ${ }^{8}$
In this study, we found only a $10 \%$ adherence rate to medication-based treatment after 6 months of follow-up. Nevertheless, only 10 patients (16\%) required surgical treatment. The remaining 50 individuals (83\%) did not request surgery even though they were made aware of this possibility. This may reflect the psychological factors associated with this condition. The concern of the patients regarding the pain is usually solved by a careful explanation of the clinical characteristics of the chronic pain and by ruling out etiologies such as neoplasia that may carry a greater psychological impact.

Nevertheless, when no improvement is experienced in the case of patients who suffer from considerable testicular discomfort, surgical treatment can be beneficial. Several surgical techniques, such as epididymectomy, ${ }^{2,9}$ vasovasostomy ${ }^{3}$ and orchiectomy, ${ }^{1}$ have been described with the objective of providing definitive relief for CTP. However, the results were not very significant until the advent of spermatic cord denervation. ${ }^{5,6,10}$ Selective microsurgical transection may result in lasting relief from CTP, as the pain in that region is mediated by the scrotal and spermatic branches of the genitofemoral and ilioinguinal nerves, as well as by sympathetic fibers that accompany the testicular artery.

Previous studies using this technique have shown success rates varying from 71 to $96 \%,{ }^{4,5,6,7,10}$ There has been one recent long-term study that evaluated 79 patients and performed this technique with a mean follow-up of 20.3 months. The study demonstrated similar results: $71 \%$ of the surgeries were successful, $17 \%$ of patients experienced partial pain relief and $12 \%$ showed no pain relief. ${ }^{10}$ In our study, $70 \%$ of the patients were cured and $20 \%$ reported improvements in pain levels. Both of our patient populations no longer use any analgesic medications, even two years after the procedure.

A majority of CTP cases can be resolved with careful orientation of the patient regarding the pain and with appropriate propaedeutic assistance. For patients who encounter great discomfort and for those who have already undergone multiple unsuccessful treatments, the microsurgical testicular denervation method may represent a promising approach.

\section{CONCLUSION}

Microsurgical testicular denervation may be a useful technique for treating severe cases of refractory CTP. In our study, it was associated with a success rate of $90 \%$. The procedure allows patients to discontinue long-term medications, and alleviates the physical and psychological suffering associated with this condition. 


\section{REFERENCES}

1. Davis BE, Noble NJ, Weigel JW, Foret JD, Mebust WK. Analysis and management of chronic testicular pain. J Urol. 1990;143:936-9.

2. Padmore DE, Norman RW, Millard OH. Analyses of indications for and outcomes of epididymectomy. J Urol. 1996;156:95-6.

3. Nangia AK, Myles JL, Thomas AJ. Vasectomy reversal for the postvasectomy pain syndrome: a clinical and histological evaluation. J Urol. 2000;164:1939-42.

4. Heidenreich A, Olbert P, Engelmann UH. Management of chronic testalgia by microsurgical testicular denervation. Eur Urol. 2002;41:3927.

5. Levine LA, Matkov TG. Microsurgical denervation of the spermatic cord as primary surgical treatment of chronic orchialgia. J Urol. 2001;165:1927-9.
6. Cadeddu JA, Bishoff JT, Chan DY, Moore RG, Kavoussi LR, Jarrett TW. Laparoscopic testicular denervation for chronic orchalgia. J Urol. 1999;162:733-6.

7. Choa RG, Swami KS. Testicular denervation. A new surgical procedurefor intractable testicular pain. Br J Urol. 1992;70:417-9.

8. Ahmed I, Rasheed S, White C, Shaikh NA. The incidence of postvasectomy chronic testicular pain and the role of nerve stripping (denervation) of the spermatic cord in its management. Br J Urol. 1997;79:269-70.

9. West AF, Leung HY, Powell PH. Epididymectomy is an effective treatment for scrotal pain after vasectomy. BJU Int. 2000;85:1097-9.

10. Strom KH, Levine LA. Microsurgical denervation of the spermatic cord for chronic orchialgia: long-term results from a single center. J Urol. 2008;180:949-53. 